\title{
March Editorial
}

Welcome to one of our most voluminous issues! Our main objective this month is to catch up with pending submissions and reduce the time between online and print publication. We have organized the issue into clusters of related areas. Some of the areas we focus on in this issue include orthopedics, tissue engineering, blood, cardiovascular, imaging, neurological, respiratory, and signal processing. We also highlight seven position papers, accompanied by an editorial by Shmuel Einav, that emerged from the Fifth International Biofluid Mechanics Symposium, held March 28-30, 2008. The symposium evaluated the state of the art of biofluid mechanics and identified new areas and challenges facing the biofluids community. We are particularly excited to include in this issue the lectures given at the 2009 BMES Annual Meeting by Sheldon Weinbaum, winner of the first annual Diversity Award, William Hawkins, winner of the Distinguished Achievement Award, and Donald Ingber, winner of the Pritzker Distinguished Lecturer award.

Although the Annals have been under the editorial leadership of the Davis office for only four months, we have implemented some significant changes. With the help of an outstanding Board of Associate Editors, the average turnaround time for manuscripts is less than four weeks. To show our appreciation, the name of the Associate Editor who oversaw the review process of a manuscript shows up now on the cover page of the published paper. We hope to have the first decision for most manuscripts within one month of submission. To do so, we would like to enlist your help by providing peer reviews in a timely manner. We depend upon the efforts of our dedicated reviewers to help us meet this goal. Let us remember that our collective objective is to elevate our journal to one of the best in biomedical engineering and bioengineering. We would also like to let authors know that we prefer to receive manuscripts that do not exceed 20 doublespaced pages, including references, but not figures. We strive to deliver issues composed of the most rigorous and cited papers that elicit wide interest. Thank you for helping us make tremendous progress. 